\title{
GESTIÓN DE LA CALIDAD EN UN GAD MUNICIPAL: UN MODELO PARA SU APLICACIÓN
}

\section{QUALITY MANAGEMENT IN A MUNICIPAL GAD: A MODEL FOR ITS APPLICATION}

\author{
Juan Diego Ochoa Crespo, Mgs. \\ Magíster en Gestión Empresarial (Ecuador). \\ Docente de la Universidad Católica de Cuenca, Ecuador. \\ jdochoac@ucacue.edu.ec \\ Cristian Manuel Ochoa Crespo, Ing. \\ Ingeniero Empresarial (Ecuador). \\ Docente de la Universidad Católica de Cuenca, Ecuador. \\ cristian.ochoa@ucacue.edu.ec
}

Jorge Edwin Ormaza Andrade, Ing.

Magíster en Administración de Empresas mención

en Recursos Humanos y Marketing (Ecuador).

Docente de la Universidad Católica de Cuenca, Ecuador.

jormaza@ucacue.edu.ec

Carlos Fabián Ramírez Valarezo, Mgs. Máster en Administración de Empresas (Ecuador). Docente de la Universidad Católica de Cuenca, Ecuador. framirezva@ucacue.edu.ec

ARTÍCULO DE REFLEXIÓN

Recibido: 12 de septiembre 2019.

Aceptado: 20 de noviembre de 2019.

ISSN: 1390-9320, Edición Especial, diciembre 2019 


\section{RESUMEN}

El presente artículo analiza la gestión de la calidad en instituciones públicas en Ecuador, específicamente en los Gobiernos Autónomos Descentralizados Cantonales (GAD), en aras de establecer un modelo que permita hacer frente a problemáticas comunes. Desde la perspectiva metodológica, se parte de un rastreo bibliográfico a partir del año 2013 hasta el año 2018 en el contexto de América Latina, en bases de datos especializadas, con la finalidad de identificar elementos vinculados con la aplicación de modelos de gestión de la calidad enfocado a instituciones del sector público. La idea de todo esto es encontrar elementos que permitan conformar un modelo de gestión de calidad que permitan alcanzar una certificación de calidad en el ámbito público. En consecuencia, se estudiaron 4 modelos de gestión de la calidad, a partir de los cuales se estableció una propuesta que recoge una serie de indicadores que permiten medir el nivel de la calidad en este tipo de instituciones enfocado a la satisfacción plena de las necesidades de la sociedad.

Palabras clave: Gestión de calidad, modelo de calidad, normas de calidad, instituciones públicas

\section{ABSTRACT}

This article analyzes the management of quality in public institutions in Ecuador, specifically in the Autonomous Decentralized Cantonal Governments (GAD), in order to establish a model that allows facing common problems. From the methodological perspective, a bibliographic search is started from 2013 until 2018 in the context of Latin America, in specialized databases, in order to identify elements linked to the application of quality management models focused on public sector institutions. The idea of all this is to find elements that make it possible to create a quality management model that allows achieving a quality certification in the public sphere. Consequently, four quality management models were studied, from which a proposal was established that includes a series of indicators that allow measuring the level of quality in this type of institutions focused on satisfying the needs of the entire population. the society.

Keywords: Quality management, quality model, quality standards, public institutions 


\section{INTRODUCCIÓN}

Una problemática común en Ecuador, es la carencia de un modelo que permita llevar a cabo de manera orientada y sistemática la gestión de la calidad en entidades del sector público, con miras de obtener una certificación que sea cónsona con los procesos desarrollados. Por ello, es pertinente analizar la gestión de calidad, apoyados en diferentes postulados a nivel mundial.

En Ecuador, de acuerdo con la Ley de Transparencia, las organizaciones del sector público están obligadas a rendir cuentas de su labor de manera periódica, esto no refleja la verdadera calidad y aplicación adecuada de los procesos. Es por ello que, (Torres, Torres, y Martínez, 2017), consideran importante medir el Índice de Transparencia Municipal, a través de indicadores claves, basado en la norma, y la verdadera satisfacción de las necesidades del entorno, desde una óptica administrativa y no política.

Por su parte, (Mel, 2015) manifiesta que, en toda entidad tanto del sector público como privado, existe una serie de deficiencias que derivan de la falta de un diseño operacional en los servicios que ofrece la institución, y por ende existe un desconocimiento del grado de satisfacción que poseen los usuarios, una falta de procesos estandarizados y normados, danto como resultado, el rompimiento de los canales entre la entidad y los beneficiarios.

Una herramienta que contribuye al manejo de la gestión de calidad de manera sistemática dentro de una organización, es la tecnología de la información. Es así que, su uso agrega valor y facilita el manejo equilibrado del riesgo dentro de los procesos, por ello es conveniente que se implementen y se evalúen una serie de reglas que contribuyan a la gestión óptima (Marulanda, Trujillo, y Valencia, 2017)

Otro elemento atado a la búsqueda de un servicio de calidad y que forma parte de este estudio, es la innovación, la cual, según Vidal (2016) tiene dos aristas; la primera, que se incluye en la gestión global de la entidad basada en la motivación y desarrollo del personal; y la segunda, que se refiera a la reivindicación del concepto burocrático de la administración pública que se adoptó en América Latina y Europa durante muchos años, hacia una transición positiva en búsqueda de la certificación de calidad con un enfoque de mejora continua. 
De lo anteriormente expuesto, se puede agregar que para la aplicación de procesos que le permitan a un GAD Municipal en Ecuador alcanzar su eficiencia en la totalidad de las operaciones, es conveniente indagar sobre la serie de modelos de gestión de calidad que existen desde lo teórico. Sobre este marco, uno de los modelos es, el modelo de la Gestión de Calidad Total (TQM), el cual, según Juanes y Blanco (2001) persigue que la entidad satisfaga las necesidades de los usuarios, a partir de sus expectativas, de una manera debidamente equilibrada, como parte de la filosofía misma de una organización.

En consecuencia, el presente artículo de revisión bibliográfica se enfoca en una serie de postulados que permiten dar una visión del concepto de calidad, y así poder ajustarlo hacia la gestión pública en Ecuador. De manera concreta, se aborda una propuesta aplicable a los Gobiernos Autónomos Descentralizados Municipales de este país.

La presente propuesta de investigación surge de la necesidad inminente que poseen las entidades del sector público en Ecuador, sobre la falta de modelos que permitan aplicar procesos adecuados de gestión de calidad, que den a conocer los costos de la no aplicación de dichos sistemas, ya que no existen investigaciones realizadas sobre este tema, en Ecuador. Por ello, la investigación tiene como objetivo inicial analizar la gestión de la calidad en instituciones públicas, a partir de los postulados teóricos vinculados a la gestión de la calidad en entidades del sector público a nivel mundial y nacional.

\section{REVISIÓN TEÓRICA}

\section{Gestión de la calidad}

(González, Mera, y Lacaba, 2007, p. 7) concibe a la calidad como la articulación armónica de todos los actores de la empresa, con la finalidad de lograr el mejor servicio posible, todo ello mediante la definición adecuada de los procesos. El mismo autor manifiesta que, la calidad debe poseer variables bien especificadas, y éstas, deberán medirse con la finalidad de cuantificar el nivel de cumplimiento mediante indicadores de eficiencia. Esto se evidencia claramente en la satisfacción de las necesidades del usuario, y en la superación de sus expectativas.

La calidad es un proceso que sigue una serie de paso o etapas bajo el criterio de calidad total, y que tiene como punto de partida el conocer la opinión del usuario, con la finalidad de saber sus expectativas y lo que piensa acerca de los procedimientos y la atención que 
le ofrece la entidad. Otro de los pasos es el manejo de la imagen de la calidad, es decir que, la organización debe filtrar la calidad de su servicio con la finalidad de que, al traspasar la frontera de la institución, ya el entorno conocerá cual es el nivel de la misma, para ello, se deben ir controlando cada proceso a medida que se van desarrollando mediante la adecuación de dichos procesos en función a estándares de calidad debidamente definidos. El horizonte siempre debe ser la plena satisfacción del usuario (Santos, 1996, p. 3)

Tomando como referencia a la norma ISO 8402, la cual sugiere que la verdadera calidad es aquella que se basa en la satisfacción plena de las necesidades del usuario de un producto o servicio, a partir de las características idóneas de dicho producto o servicio. Por ello, es pertinente que, se enfoque este concepto de calidad hacia varios aspectos: un primer aspecto es, el significado que tiene la calidad para el cliente o usuario, esto se logra mediante el estudio de las necesidades de quien acude a la organización, ya que este no cumple el único proceso de adquirir un producto o gozar de un servicio, sino al contrario, es el hecho de trascender como organización hacia la satisfacción plena de una necesidad, para ello deben darse las facilidades correspondientes para que el usuario pueda ser beneficiario del servicio. En este camino, la empresa debe medir de manera constante el grado de satisfacción del cliente, para conocer si se cumplen o no las expectativas. Otro de los elementos claves para el cumplimiento de un sistema de gestión de la calidad, lo constituye la automatización de procesos, es decir, el uso de la mejor tecnología para la implementación eficiente de cada acción, lo que implica que, se analicen los gastos innecesarios, y se potencien únicamente los requerimientos económicos que vayan a contribuir al concepto de calidad (Rey, 2011, pp. 1-3).

En la siguiente tabla se resume la evolución concepto de calidad, sus características, sus objetivos, orientaciones, implantación y métodos:

Tabla 1 Evolución del concepto de calidad

\begin{tabular}{|c|c|c|c|c|}
\hline & Objetivos & Orientación & Implantación & Métodos \\
\hline $\begin{array}{l}\text { Gestión de la } \\
\text { calidad total }\end{array}$ & $\begin{array}{l}\text { Impacto } \\
\text { estratégico }\end{array}$ & $\begin{array}{l}\text { Satisfacción } \\
\text { plena del } \\
\text { cliente }\end{array}$ & $\begin{array}{l}\text { Toda la } \\
\text { organización }\end{array}$ & $\begin{array}{l}\text { Planificación } \\
\text { estratégica }\end{array}$ \\
\hline
\end{tabular}




\begin{tabular}{|c|c|c|c|c|}
\hline $\begin{array}{l}\text { Control del } \\
\text { proceso }\end{array}$ & $\begin{array}{l}\text { Organización y } \\
\text { coordinación }\end{array}$ & $\begin{array}{l}\text { Aseguramiento } \\
\text { y prevención }\end{array}$ & $\begin{array}{l}\text { Dpto. de } \\
\text { Calidad } \\
\text { Producción, } \\
\text { I+D }\end{array}$ & $\begin{array}{l}\text { Sistemas, } \\
\text { técnicas } \\
\text { programas }\end{array}$ \\
\hline $\begin{array}{l}\text { Control del } \\
\text { producto }\end{array}$ & $\begin{array}{l}\text { Control de } \\
\text { productos }\end{array}$ & $\begin{array}{l}\text { Reducción de } \\
\text { inspecciones }\end{array}$ & $\begin{array}{l}\text { Departamento } \\
\text { de calidad }\end{array}$ & $\begin{array}{l}\text { Muestreo } \\
\text { estadísticas }\end{array}$ \\
\hline Inspección & $\begin{array}{l}\text { Detección de } \\
\text { defectos }\end{array}$ & $\begin{array}{l}\text { Orientación al } \\
\text { producto }\end{array}$ & $\begin{array}{l}\text { Departamento } \\
\text { de inspección }\end{array}$ & $\begin{array}{l}\text { Medición } \\
\text { verificación }\end{array}$ \\
\hline
\end{tabular}

Fuente: López Rey (2011)

Como se puede observar en la tabla anterior, el concepto de calidad desde su origen, tuvo como objetivo la detección de defectos, con una orientación hacia el producto, para lo cual la medición y verificación era llevada a cabo por el departamento de inspección. Luego, se introdujo el concepto de control del producto, reduciendo las inspecciones y llevando a cabo procesos de muestreo y de búsqueda de información estadística. Posterior a esta concepción, surgió el control del proceso, con el objeto de organizar y coordinar el aseguramiento de la calidad y la prevención, a través del departamento de calidad, producción, investigación y desarrollo; mediante el uso de sistemas, técnicas y programas. Finalmente, la concepción de calidad ha llegado a tener un enfoque conocido como gestión de la calidad total, como un impacto estratégico orientado hacia la satisfacción plena del cliente, involucrando a toda la organización, mediante una planificación estratégica adecuada.

\section{Modelos de gestión de calidad}

Luego de la revisión de los siguientes autores: (González y Rodríguez, 2008); (Rey, 2011); (Mancera, 2013); (González, Maldonado, P, y Derlin Ramírez Z, 2013); (Cárdenas y García Treviño, 2014); (Camejo, 2014); (Torres, 2014); (Saenz y Palomino, 2014); (Ugarte, 2015); (Bretaña, Valdés, y González, 2015); (Pont, 2016); (Acuña, Romero, y López, 2016); (Penagos y Fonseca, 2016); (Navarro y Malta, 2016); (Guasch, Tovio, y Mangones, 2016); (Dueñas, Godoy, \& Rodríguez, 2016); (Jiménez, 2016); (Alfonso, Acevedo, Ramírez, y Rodríguez, 2016); (Torres, Torres, y Martínez, 2017); (Palma, Arbeláez, y Carreño, 2017); (Echeverry y Trujillo, 2017); (Vargas, 2017); se seleccionaron los siguientes modelos 
referenciales en función de las unidades de análisis porque son aplicables a instituciones del sector público.

(Juanes y Blanco, 2001) ilustra en el siguiente gráfico la articulación del sistema de gestión de calidad total:

Gráfico. 1 Sistemas de gestión de calidad total

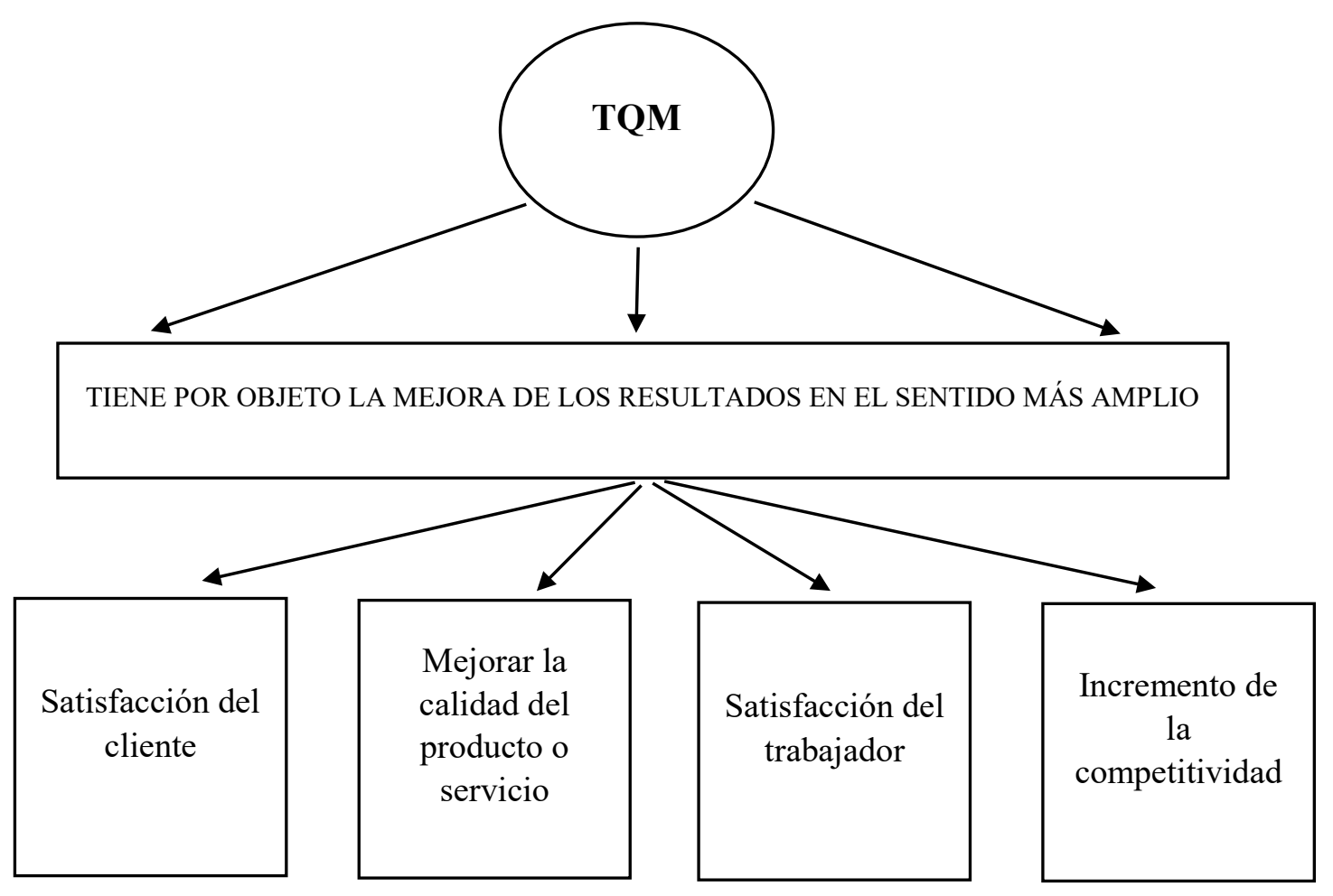

Fuente: Elaboración propia a partir de Bruno; Juanes 2001.

Según lo ilustra (González y Rodríguez, 2008), el modelo de gestión de calidad con un enfoque de ingeniería de calidad es una alternativa muy valiosa para lograr la eficiencia en una organización, ya que este parte de la creación de equipos de trabajo para cada proceso de la empresa, donde se definen claramente las funciones y roles de cada uno de los miembros; seguidamente este modelo propone, identificar las necesidades del cliente, para lo cual, se debe contar con una lista de usuarios debidamente clasificados, con la finalidad de elaborar un mapa de procesos interrelacionados. Luego el modelo sugiere definir los objetivos y políticas para delimitar las estrategias; de la cual se deben derivar las 
herramientas claramente establecidas, diseñar los formatos para la elaboración de los diferentes tipos de documentos, se aprueban los mismos. Finalmente, se deben desarrollar las actividades con la debida supervisión a partir de auditorías y la medición de resultados por medio de indicadores.

Un modelo genérico de sistema de gestión de calidad debe tener tres componentes básicos: primero se requiere un levantamiento de un mapa de procesos, mediante la diagramación y caracterización debiendo definirse procesos, actividades, documentos requeridos, responsables, indicadores y requisitos; seguidamente, es pertinente que la empresa defina sus objetivos y delimite su plan de acción para lograrlos, aquí se deben definir responsables y asignar recursos presupuestarios; por último, se debe estar pendiente de los cambios en el entorno, y principalmente en los cambios en las normas y procedimientos por parte de los órganos de control, todo esto con la finalidad de estar ajustándose continuamente al entorno (Hurtado, 2005, p. 49).

Otro modelo que es aplicable a un GAD Municipal en Ecuador, lo constituye el llamado REDER: Resultados, Enfoque, Despliegue, Evaluación y Revisión, que se resume en la siguiente tabla:

Tabla 2. Modelo REDER de gestión de la calidad

\begin{tabular}{|c|l|}
\hline \multicolumn{2}{|c|}{ Agentes } \\
\hline $\begin{array}{c}\text { Criterio 1. } \\
\text { Liderazgo }\end{array}$ & $\begin{array}{l}\text { Cómo el equipo directivo concibe y dirige la calidad total como } \\
\text { proceso fundamental de la empresa para la mejora continua. }\end{array}$ \\
\hline $\begin{array}{c}\text { Criterio 2. } \\
\text { Política y estrategia }\end{array}$ & $\begin{array}{l}\text { Cómo la organización implanta su misión y visión mediante una } \\
\text { estrategia claramente centrada en todos los grupos de interés y } \\
\text { apoyada por políticas, planes, objetivos, metas y procesos } \\
\text { relevantes. }\end{array}$ \\
\hline $\begin{array}{l}\text { Criterio 3. } \\
\text { Personas }\end{array}$ & $\begin{array}{l}\text { Cómo la organización gestiona, desarrolla y aprovecha el } \\
\text { conocimiento y todo el potencial de las personas que la } \\
\text { componen, tanto en el ámbito individual, como de equipos o de } \\
\text { la organización en su conjunto, planificando estas actividades en } \\
\text { apoyo de su política y estrategia y del eficaz funcionamiento de } \\
\text { sus procesos. }\end{array}$ \\
\hline
\end{tabular}

ISSN: 1390-9320, Edición Especial, diciembre 2019 


\begin{tabular}{|c|c|}
\hline $\begin{array}{l}\text { Criterio } 4 \text {. } \\
\text { Alianzas y recursos }\end{array}$ & $\begin{array}{l}\text { Cómo la organización planifica y gestiona sus alianzas externas } \\
\text { y recursos internos en apoyo de su política y estrategia y del } \\
\text { eficaz funcionamiento de sus procesos. }\end{array}$ \\
\hline $\begin{array}{l}\text { Criterio } 5 . \\
\text { Procesos }\end{array}$ & $\begin{array}{l}\text { Cómo la organización diseña, gestiona, y mejora sus procesos } \\
\text { para apoyar su política y estrategia para satisfacer plenamente, } \\
\text { generando cada vez mayor valor a sus clientes y otros grupos } \\
\text { de interés. }\end{array}$ \\
\hline \multicolumn{2}{|r|}{ Resultados } \\
\hline $\begin{array}{c}\text { Criterio } 6 . \\
\text { Resultados en los } \\
\text { clientes / } \\
\text { Satisfacción del } \\
\text { cliente }\end{array}$ & $\begin{array}{l}\text { Cuáles son los logros que la organización está alcanzando en } \\
\text { relación con la satisfacción con sus clientes externos. }\end{array}$ \\
\hline $\begin{array}{c}\text { Criterio } 7 . \\
\text { Resultados en los } \\
\text { procesos / } \\
\text { Satisfacción del } \\
\text { personal }\end{array}$ & $\begin{array}{l}\text { Cuáles son los logros que la organización está alcanzando en } \\
\text { relación con la satisfacción de las personas que la integran. }\end{array}$ \\
\hline $\begin{array}{l}\text { Criterio } 8 \text {. } \\
\text { Resultados en la } \\
\text { sociedad / Impacto } \\
\text { en la sociedad }\end{array}$ & $\begin{array}{l}\text { Cuáles son los logros de la organización en cuanto a la } \\
\text { satisfacción de las necesidades y expectativas de la comunidad } \\
\text { en general, incluyendo tanto opiniones acerca de la orientación } \\
\text { de la empresa, hacia la calidad de vida, el medio ambiente y la } \\
\text { conservación global de recursos naturales, como las propias } \\
\text { mediciones de la empresa. }\end{array}$ \\
\hline $\begin{array}{c}\text { Criterio } 9 . \\
\text { Resultados } \\
\text { empresariales }\end{array}$ & $\begin{array}{l}\text { Cuáles son los logros de la organización en relación al } \\
\text { rendimiento económico planificado, y con la satisfacción de las } \\
\text { necesidades y expectativas de todos aquellos que tengan } \\
\text { intereses financieros en la empresa. }\end{array}$ \\
\hline
\end{tabular}

Fuente: López Rey (2011)

ISSN: 1390-9320, Edición Especial, diciembre 2019 


\section{La norma ISO 9001:2015}

La Organización Internacional de Normalización (ISO), constituye una federación a nivel mundial que tiene la finalidad de preparar las normas que orientan el accionar de las organizaciones para el cumplimiento de un objetivo específico. Sobre este marco, las empresas como parte de su delimitación de estrategias, pueden adoptar modelos de gestión de la calidad. La norma ISO 9001:2015 es completamente aplicable a una institución del sector público, de ahí que en un GAD Municipal, es pertinente tener un enfoque hacia los procesos, teniendo como horizonte la satisfacción plena de las satisfacciones del cliente o usuario, a partir de sus intereses y requisitos.

En la siguiente tabla se delimitan algunas características de este modelo:

Tabla 3 Características de la norma ISO 9001:2015

\begin{tabular}{|c|c|}
\hline Elementos & Características \\
\hline $\begin{array}{l}\text { Contexto de la } \\
\text { organización }\end{array}$ & $\begin{array}{l}\text { La organización debe hacer un análisis de su } \\
\text { entorno, a partir del cual identifique sus fortalezas, } \\
\text { debilidades, oportunidades y amenazas, a partir delas } \\
\text { cuales deberá formular sus objetivos y delimitar sus } \\
\text { estrategias. } \\
\text { - En este proceso, la organización debe identificar } \\
\text { claramente a los grupos de interés, así como conocer } \\
\text { cuáles son las demandas de estos grupos en aras de } \\
\text { lograr la calidad del servicio. } \\
\text { - Como todo sistema posee entradas y salidas, en } \\
\text { esta parte, se deben conocer los recursos con los que se } \\
\text { cuenta para la aplicación de procesos, considerando el } \\
\text { elemento de salida, que en este caso sería el servicio de } \\
\text { calidad. } \\
\text { - } \\
\text { medición, y la asignación de los responsables para cada } \\
\text { actividad. }\end{array}$ \\
\hline
\end{tabular}

ISSN: 1390-9320, Edición Especial, diciembre 2019 


\begin{tabular}{|l|l|}
\hline & $\begin{array}{l}\text { Otro elemento que forma parte del contexto de la } \\
\text { organización es el análisis y medición del riesgo, para lo } \\
\text { cual se deben tener documentos de sustento. }\end{array}$ \\
\hline & La organización debe contar con un responsable \\
de monitorear el cumplimiento del modelo de calidad, y \\
rendir cuentas sobre ello. Para que esto se pueda llevar \\
a cabo, es pertinente tener claramente definido lo \\
siguiente: la política de la calidad, objetivos claros de la \\
calidad, los requisitos del sistema de la calidad con \\
enfoque de procesos. \\
- El responsable de la institución debe estar \\
comprometido para ofrecer todo el apoyo al equipo de \\
trabajo, facilitando los roles de cada uno de los \\
miembros. \\
Liderazgo \\
cliente, con la finalidad de asegurar la plena satisfacción \\
del mismo. \\
- El director de la entidad debe tener claramente \\
delimitada la política de calidad y garantizar su \\
aplicación, con la filosofía de mejoramiento continuo. \\
L La política de calidad debe ser informada y \\
socializada, al mismo tiempo se deben capacitar sobre \\
la misma, a todo el personal que involucra la \\
organización.
\end{tabular}




\begin{tabular}{|c|c|}
\hline & $\begin{array}{l}\text { - Los objetivos deben tener responsables y al } \\
\text { mismo tiempo deben contar con una asignación } \\
\text { presupuestaria. } \\
\text { - En el caso de existir un cambio en el sistema de } \\
\text { gestión de la calidad, es pertinente elaborar el plan de } \\
\text { contingencia frente a dichos cambios, y socializarlos a } \\
\text { toda la organización. }\end{array}$ \\
\hline Ароуо & $\begin{array}{l}\text { - No se puede llevar a cabo un proceso de gestión } \\
\text { de la calidad, si no se cuenta con el apoyo financiero } \\
\text { correspondiente para el mismo. Para este fin, seden } \\
\text { conocer las limitaciones y alcances en el marco } \\
\text { presupuestario. } \\
\text { - El recurso humano es el elemento fundamental } \\
\text { para la implementación de un sistema de gestión de la } \\
\text { calidad. } \\
\text { - } \\
\text { clave para la implementación de un sistema de gestión } \\
\text { la calidad, desde los siguientes aspectos: edificaciones, } \\
\text { equipo de cómputo, transporte, tecnologías de la } \\
\text { información, etc. } \\
\text { - El ambiente laboral en el desarrollo de procesos } \\
\text { de calidad debe considerar: solución de conflictos, } \\
\text { inclusión, aspectos psicológicos de los empleados, área } \\
\text { física, etc. } \\
\text { - } \\
\text { debe ser de calidad y este requiere un monitoreo } \\
\text { permanente. } \\
\text { - } \text { La capacitación y actualización de conocimientos } \\
\text { niveles. } \\
\text { realizar un análisis del personal, ya que es fundamental }\end{array}$ \\
\hline
\end{tabular}




\begin{tabular}{|c|c|}
\hline & $\begin{array}{l}\text { que cada persona posea las competencias necesarias } \\
\text { para el cargo que desempeña. } \\
\text { - El personal debe tomar conciencia de lo } \\
\text { fundamental que es la política de calidad y contribuir a } \\
\text { su aplicación. } \\
\text { - La política de comunicación tanto interna como } \\
\text { externa de la entidad debe considerar: qué comunicar, } \\
\text { cuándo comunicar, a quién comunicar, cómo comunicar, } \\
\text { qué comunicar. } \\
\text { - Todos los procesos y acciones deben ser } \\
\text { debidamente documentados, para ello siempre se deben } \\
\text { usar canales formales. Para ello, la documentación debe } \\
\text { estar bien ordenada considerando: título, fecha, } \\
\text { pertenencia, autor, etc. En este elemento, es pertinente } \\
\text { que una comisión evalúe de manera periódica la } \\
\text { documentación institucional, la cual debe contar con el } \\
\text { espacio físico adecuado. (En esta parte es fundamental } \\
\text { implementar políticas encaminadas a la digitalización de } \\
\text { la documentación) }\end{array}$ \\
\hline Operación & $\begin{array}{l}\text { - La comunicación al cliente debe proporcionar } \\
\text { información completa del producto o servicio, obtener } \\
\text { retroalimentación del mismo, y dar una atención } \\
\text { posterior a la venta o prestación del servicio. } \\
\text { - } \quad \text { Loa productos tienen requisitos reglamentarios. } \\
\text { - } \quad \text { Los productos o servicios deben estar ajustando } \\
\text { a los cambios del entorno y la competencia. } \\
\text { - } \quad \text { Los procesos deben estar claramente diseñados } \\
\text { considerando sus etapas e indicadores de medición. } \\
\text { - } \quad \text { Los clientes son la voz activa y participativa en la } \\
\text { delimitación de procesos y prestación del servicio. } \\
\text { - } \quad \text { El producto o servicio debe estar sujeto a las } \\
\text { normas correspondientes que los regulan. }\end{array}$ \\
\hline
\end{tabular}




\begin{tabular}{|c|c|}
\hline & $\begin{array}{l}\text { - } \text { El control debe garantizar el cumplimiento } \\
\text { adecuado de los procesos. } \\
\text { - } \quad \text { La calidad debe medirse en base a indicadores. } \\
\text { - } \quad \text { La relación con los proveedores es activa. Estos } \\
\text { deben conocer procesos, documentos, y transparencia. } \\
\text { - La organización debe velar por mantener a sus } \\
\text { clientes estableciendo procesos que persigan la fidelidad } \\
\text { de los mismos. } \\
\text { - } \quad \text { Los procesos deben tener contenido ambiental, } \\
\text { tanto desde lo administrativo interno, como en lo } \\
\text { referente al servicio que se dan en forma externa. } \\
\text { - En el caso de existir inconsistencias en el servicio } \\
\text { prestado, la organización está en la obligación de } \\
\text { corregir o recompensar por el servicio ineficiente. Por } \\
\text { ello, debe contar con una política de devoluciones y su } \\
\text { proceso claramente definido. }\end{array}$ \\
\hline $\begin{array}{c}\text { Evaluación del } \\
\text { desempeño }\end{array}$ & $\begin{array}{l}\text { - Es necesario evaluar el grado de satisfacción del } \\
\text { cliente, y darle un valor muy grande al mismo. } \\
\text { - } \quad \text { Antes de que los organismos de control apliquen } \\
\text { auditorías en las instituciones, estas deben tener una } \\
\text { política de auditoría interna, para garantizar el } \\
\text { cumplimiento adecuado de los procesos. } \\
\text { - } \quad \text { Todos los niveles de la organización forman parte } \\
\text { de la evaluación de desempeño. } \\
\text { - } \quad \text { La empresa debe tener bien planificado el } \\
\text { conjunto de actividades que permitan corregir las } \\
\text { inconformidades dentro del proceso de medición de } \\
\text { desempeño. } \\
\text { - } \\
\text { directivos indispensable la predisposición de los altos } \\
\text { necesario, dependiendo del nivel de desempeño. }\end{array}$ \\
\hline
\end{tabular}




\begin{tabular}{|l|l|l|}
\hline \multirow{3}{*}{ Mejora } & La empresa debe mejorar los productos \\
& constantemente, atendiendo a las necesidades del \\
& usuario y el entorno. \\
& En caso de existir indicadores de calidad \\
& eficientes, igualmente se debe seguir buscando la \\
& mejora continua del servicio.
\end{tabular}

Fuente: Elaboración propia a partir de la norma ISO 9001:2015

\section{Propuesta teórica}

La descentralización constituye un concepto que se utiliza actualmente para referirse a los gobiernos seccionales en Ecuador, es así que, dependiendo de las competencias, la Constitución de la República del Ecuador los divide en: Gobiernos Regionales, Gobiernos Provinciales, Distritos Metropolitanos, Gads Municipales, y Gads Parroquialies. Un Gobierno Autónomo Descentralizado (GAD) Municipal, es un gobierno seccional, responsable de ejecutar lo establecido en la ley, con la finalidad de garantizar el bienestar de la población para la cual ofrece su servicio y lleva a la práctica su gestión. Los principios que rigen el accionar de los Gads, se encuentran estipulados en el Código Orgánico de Organización Territorial, Autonomía y Descentralización (COOTAD), mismo que define los ejes de gestión principales: gestión de servicios y aplicación de políticas públicas; equidad, justicia y redistribución; recaudación; transparencia mediante la rendición de cuentas periódica; regulación del territorio; dotación de servicios básicos (Gómez, 2013)

En atención a las competencias de un GAD Municipal, se analizaron varios modelos de acuerdo con la afinidad de las mismas y los intereses del autor, de tal manera que en la siguiente tabla se evidencian los principios fundamentales que contribuyen a la correcta gestión de la calidad:

Tabla 4. Principios fundamentales por modelo

\begin{tabular}{|c|cl|}
\hline MODELO & & PRINCIPIOS FUNDAMENTALES \\
\hline \multirow{2}{*}{$\begin{array}{c}\text { Sistema de gestión de } \\
\text { calidad total }\end{array}$} & $\bullet$ & Satisfacción del cliente \\
& $\bullet$ & Sejorar la calidad del servicio \\
& $\bullet$ & Incremento de la competitividad \\
\hline
\end{tabular}

ISSN: 1390-9320, Edición Especial, diciembre 2019 


\begin{tabular}{|c|c|}
\hline $\begin{array}{l}\text { Modelo genérico de } \\
\text { gestión de la calidad }\end{array}$ & $\begin{array}{l}-\quad \text { Mapa de procesos } \\
\text { - } \quad \text { Estar en concordancia con los cambios del } \\
\text { entorno }\end{array}$ \\
\hline $\begin{array}{c}\text { Modelo REDER } \\
\text { (Resultados, Enfoque, } \\
\text { Despliegue, Evaluación y } \\
\text { Revisión) }\end{array}$ & $\begin{array}{ll}\text { - } & \text { Liderazgo } \\
\text { - } & \text { Planificación estratégica } \\
\text { - } & \text { Procesos que aprovechan las habilidades y } \\
\text { potencialidades del personal. } \\
\text { - } \quad \text { Asignación de recursos } \\
\text { - } \quad \text { Procesos enfocados al usuario y grupos de } \\
\text { interés. }\end{array}$ \\
\hline $\begin{array}{l}\text { Modelo basado en la } \\
\text { norma ISO 9001:2015 }\end{array}$ & 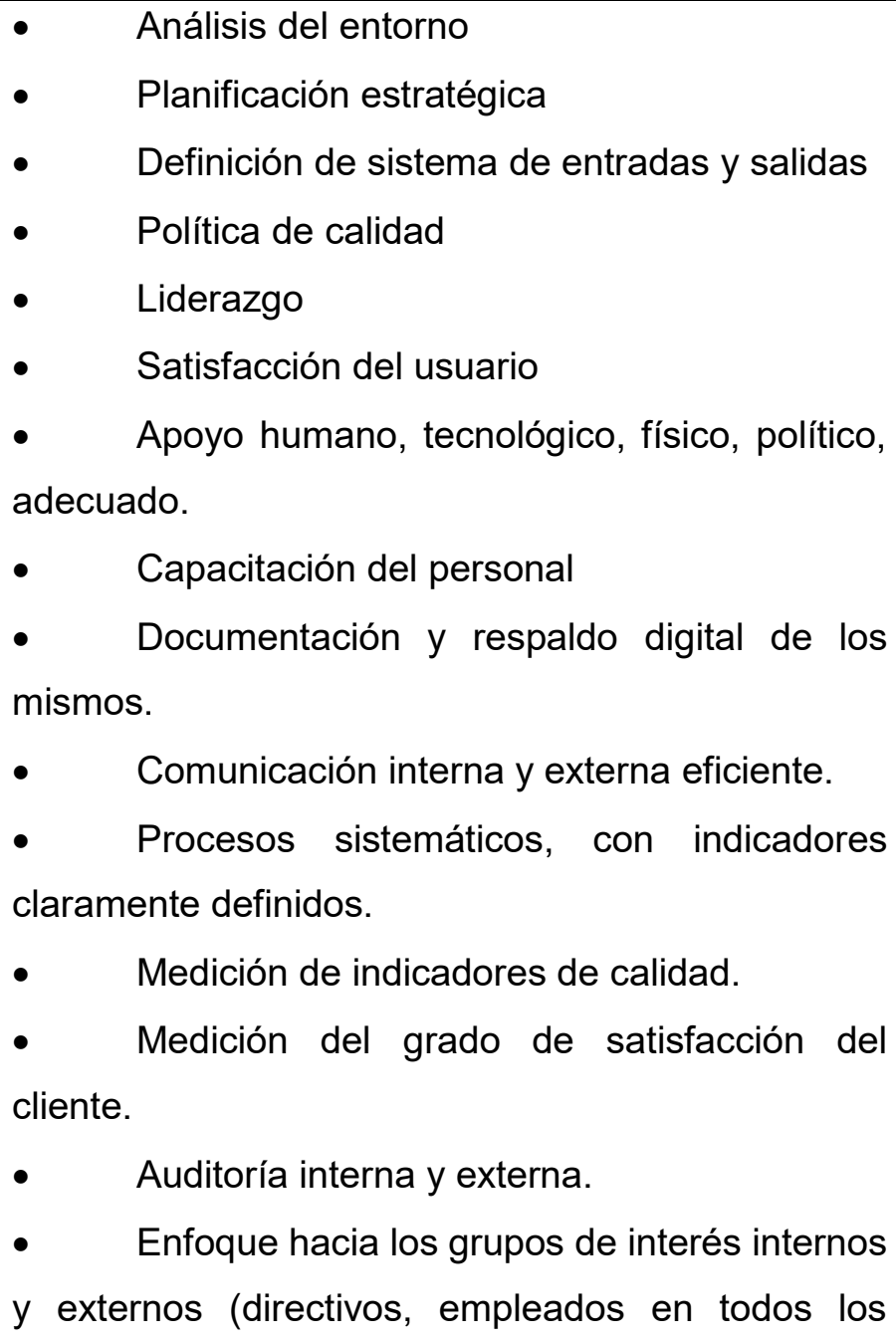 \\
\hline
\end{tabular}




\begin{tabular}{|l|l|}
\hline & $\begin{array}{l}\text { niveles, usuarios, medios de comunicación, otras } \\
\text { instituciones, etc. })\end{array}$ \\
\hline
\end{tabular}

Fuente: Elaboración propia.

\section{CONCLUSIONES}

El presente artículo tiene las siguientes conclusiones:

- Un GAD Municipal en Ecuador, tiene como sustento legal el COOTAD, sin embargo, es pertinente que los procesos se ajusten hacia la satisfacción plena de las necesidades de los usuarios, por lo cual la gestión debe ser de calidad.

- Los modelos de gestión de la calidad tienen su origen en la administración privada, pero son completamente aplicables a una institución del sector público.

- Todos los modelos de gestión de la calidad, tienen elementos en común, tales como: análisis del entorno, planificación estratégica, liderazgo, apoyo, evaluación de desempeño, mejora continua.

- La mayoría de instituciones del sector público no tienen un plan de acción a seguir en cuanto a la calidad, por ello se sugiere considerar los principios que el presente documento recomienda.

\section{REFERENCIAS BIBLIOGRÁFICAS}

Acuña, D., Romero, C., y López, D. (2016). Sistema integral de gestión de calidad en la universidad de Guajira, Colombia. Telos, 474-491.

Alfonso, Y. R., Acevedo-Suárez, J. A., Ramírez-Betenacourt, F., y Rodríguez, E. G. (2016). Modelo de gestión de la eficiencia basado en los costos de la calidad con enfoque generalizador. Ingeniería Industrial, 59-69.

Bretaña, R. M., Valdés, M. d., y González, R. (2015). Normalización y aplicación de los principios de gestión de la calidad en la actividad archivística. Revista Habanera de Ciencias Médicas, 527-535. 
Camejo, R. V. (2014). Impacto de la implantación de modelos de gestión de calidad y excelencia en la administración pública: caso de República Dominicana. Revista del CLAD Reforma y Democracia, 221-236.

Cárdenas, M. A., y García Treviño, A. H. (2014). Gestión de la calidad educativa en educación superior del sector privado. Magis. Revista Internacional de Investigación en Educación, 65-82.

Dueñas, M. H., Godoy, S. A., y Rodríguez, J. P. (2016). Sistemas de calidad y desempeño empresarial: estudio de caso en empresas cárnicas en una región del noreste de México. Ingeniería Industrial, 97-117.

Echeverry, C. E., y Trujillo, M. L. (2017). Gobierno y gestión de las TI en las entidades públicas. AD-minister, 75-92.

Gómez, H. B. (2013). El actual modelo de descentralización en el Ecuador: un desafío para los gobiernos autónomos descentralizados. Foro: Revista de Derecho.

González, A. G., \& Rodríguez, R. A. (2008). Diseño de un sistema de gestión de la calidad con un enfoque de ingeniería de la calidad. Ingeniería Industrial, 1 - 6.

González, A., Maldonado, X., P, L. N., y Derlin Ramírez Z. (2013). Evaluación de la Gestión de Calidad en la Facultad de Ciencias Económicas y Sociales. Visión Gerencia, 293-324.

González, F. M., Mera, A. C., y Lacaba, S. R. (2007). Introducción a la gestión de la calidad. Madrid: Delta, Publicaciones universitarias.

Guasch, J. C., Tovio, J. M., y Mangones, Á. P. (2016). Gestión de calidad en sistemas tecnológicos de información en las organizaciones públicas del departamento Sucre - Colombia. Espacios Públicos, 7-19.

Hurtado, F. A. (2005). Gestión y auditoría de la calidad para organizaiones públicas. Medellin, Colombia: Editorial Universidad de Antioquia .

Jiménez, L. F. (2016). Los costos de la mala calidad como quinto elemento del costo aproximación térocia en la gestión en medo de la competitivad en la 
convergencia contable. Revista Facultad de Ciencias Económicas: Investigación y Reflexión, 63-84.

Juanes, B., \& Blanco, J. (2001). Modelos de calidad en la administración pública. Ediciones Días de Santos, S.A: España.

Mancera, L. G. (2013). Mejoramiento de la gestión publica con ISO 9001:2008, estudio de caso. Scientia Et Technica, 126 - 131.

Marulanda, C. E., Trujillo, M. L., y Valencia, F. J. (2017). Gobierno y gestión de TI en las entidades públicas. AD-minister, 75 - 92.

Mel, J. U. (2015). Nueva estrategia para la gestión de calidad: implementación de cartas de servicios en una entidad pública peruana. Ingeniería industrial, 51 68.

Navarro, C. A., y Malta, N. C. (2016). Criterios para cuantificar costos y beneficios en proyectos de mejora de calidad. Ingeniería Industrial, 151-163.

Palma, H. G., Arbeláez, D. A., y Carreño, M. P. (2017). Proyeccion estratégica de la calidad como facilitadora para la innovacion en el sector salud de la ciudad de Barranquilla. Revista Lasallista de Investigación, 170-178.

Penagos, C. P., y Fonseca, F. R. (2016). La capacitación y su efecto en la calidad dentro de las organizaciones. Revista de investigación y desarrollo e innovación, 131143.

Pont, J. V. (2016). Modelos innovadores de administración y gestión pública: Hacia la emergencia de nuevos paradigmas. Gestión y Análisis de Políticas Públicas.

Rey, S. L. (2011). Sistemas de calidad. Implantación de diferentes sistemas en la organización. Bogotá, Colombia: Ediciones de la U.

Rey, S. L. (2011). Sistemas de calidad. Implantación de diferentes sistemas en la organización. Bogotá, Colombia: Ediciones de la U.

Saenz, J. M., \& Palomino, N. L. (2014). Modelo de Calidad para la Gestión de las TIC en el Proceso de Enseñanza paralnstituciones Educativas Peruanas del Nivel Secundario. Industrial Data, 110-119. 
Santos, D. d. (1996). Gestión de la calidad total. Madrid, España: Ediciones Días de Santos S. A.

Torres, C. A. (2014). Orientaciones para implementar una gestión basada en procesos. Ingeniería Industrial, 159-171.

Torres, H. A., Torres, D. A., y Martínez, R. C. (2017). Transparencia municipal método de cálculo de indicador condensado. Propuesta para los gobiernos locales de Michoacán México. Revista Enfoques: Ciencia Política y Administración Pública, $37-66$.

Torres, H. A., Torres, D. A., y Martínez, R. C. (2017). Transparencia municipal: método de cálculo de indicador condensado. Propuesta para. Revista Enfoques: Ciencia Política y Administración Pública, 37 - 66.

Ugarte, M. J. (2015). Nueva estrategia para la gestión de calidad: implementación de cartas de servicios en una entidad pública peruana. Ingeniería Industrial, 51-68.

Vargas, F. M. (2017). Los consejos económicos y sociales como promotores del diálogo. Iberofórum. Revista de Ciencias Sociales de la Universidad Iberoamericana, 166-188.

Vidal, J. P. (2016). Modelos innovadores de administración y gestión pública: Hacia la emergencia de. Gestión y Análisis de Políticas Pública. 